\title{
Long-Range Mass Transport during Structural Transitions in Metallic Glass-Forming Melts
}

\author{
I. Jonas, F. Yang, ${ }^{*}$ and A. Meyer \\ Institut für Materialphysik im Weltraum, Deutsches Zentrum für Luft- und Raumfahrt (DLR), 51170 Köln, Germany
}

(Received 16 November 2018; revised manuscript received 12 April 2019; published 2 August 2019)

\begin{abstract}
We investigate the structure and dynamics of the bulk metallic glass-forming alloys $\mathrm{Zr}_{41.2} \mathrm{Ti}_{13.8} \mathrm{Cu}_{12.5} \mathrm{Ni}_{10} \mathrm{Be}_{22.5}$ and $\mathrm{Zr}_{58.5} \mathrm{Cu}_{15.6} \mathrm{Ni}_{12.8} \mathrm{Al}_{10.3} \mathrm{Nb}_{2.8}$. Combining in situ synchrotron $\mathrm{x}-$ ray diffraction and quasielastic neutron scattering with electrostatic levitation, we directly observe an abrupt change in the temperature dependence of the first structure factor maximum of these melts. We find that the kinetics of this liquid-liquid transition during cooling are on the order of tens of seconds, whereas its onset temperature depends only weakly on the applied cooling rate. Such slow transition kinetics require longrange mass transport, which is incompatible with a transition mechanism involving only local structural changes as in oxides or molecular liquids.
\end{abstract}

DOI: 10.1103/PhysRevLett.123.055502

Phase transformation between two disordered structures, i.e., the so-called polyamorphism, is a long-standing issue in the field of liquid and glass physics, still being intensively studied and debated. This kind of liquid-liquid (LL) transition was first suggested to be present in water due to its unusual properties in the undercooled state [1]. Indications of its existence have then been continuously exhibited through experiments and simulations, for example, in oxide melts such as $\mathrm{Al}_{2} \mathrm{O}_{3}-\mathrm{Y}_{2} \mathrm{O}_{3}$, [2] $\mathrm{SiO}_{2}$ [3], semiconductors such as $\mathrm{Si}$ and $\mathrm{Ge}$ [4,5], or recently, a number of metallic glass-forming alloys [6-11]. It has been proposed that such transitions can be interpreted as analogous to an order-disorder transition, with a diverging correlation length at the transition point and a lambdashaped heat capacity [1,9]. This scenario implies large impacts on the structural and dynamic properties of the melt, including the liquid viscosity $[6,8]$ or the glassforming ability [1], and hence, it is also of interest for materials science.

However, unlike the polymorphic transition in crystalline materials, the physical nature and microscopic mechanism of such a transition is not well understood. For example, it is unclear whether such a transition is accompanied by a critical point, although the thermodynamic manifestations are rather similar $[1,12]$. Accordingly, its first-order transition nature is still under debate. For oxide and molecular liquids, the microscopic mechanism of the LL transition can be often understood structurally as a change of the bonding angle or the coordination of structural units [1-5], which involves high- and low-density phases. A typical case is the high-to-low density transition in amorphous ice, which appears to be diffusion controlled [13].

Classical metallic bonding is, however, nondirectional. In metallic glass or melts, it has been shown that the pressure-driven polyamorphic transition in the glassy state involves delocalization of the electrons, which is distinct from the change of the coordination and topological structure [7]. For many other cases, a mismatch in the high- and low-temperature-dependent melt viscosities-the so-called strong-to-fragile transition-was found, which is considered to be a LL transition driven by the temperature $[6,8,11]$. In contrast to molecular or oxide melts, changes of the medium-range order are considered to be relevant for the LL transition in alloy melts [9,14-17]. However, it is still questionable whether and how the observed strong-tofragile transition in metallic glass-forming liquids can be understood in accordance with the general picture of the LL transition together with other glass formers.

Most of the LL transitions in metallic glass-forming liquids were observed in the undercooled liquid region $[9,10,14,16]$. The direct experimental access is hence challenging, and the discussion remains largely inconclusive and controversial [2,18-20]. Only recently, combined with advanced experimental techniques, for a few alloys with excellent glass-forming abilities, consistent structural and thermodynamics features have been characterized not only for two different phases separately but also directly across the region where the LL transition occurs $[9,16,17]$. In addition to a viscosity mismatch of approximately one decade, this includes an abrupt increase in the heat capacity and a sudden change in the temperature-dependent first structural factor maximum $q_{\max }$, however, not accompanied by macroscopic density changes larger than $0.1 \%$. It has also been shown that during ultrafast heating of glassy ribbons, the transition temperature seems to depend on the heating rate [17]. This knowledge is essential to reveal the transition kinetics in order to resolve the underlying mechanisms and their thermodynamic classification. 
However, so far, only very few liquids have been investigated. More importantly, the melt is not sufficiently stable at the transition temperature to allow direct study of the dynamics, and the conclusions are still far from unambiguous.

Here we report an investigation of structure and dynamics of the bulk metallic glass- (BMG) forming melts $\mathrm{Zr}_{41.2} \mathrm{Ti}_{13.8} \mathrm{Cu}_{12.5} \mathrm{Ni}_{10} \mathrm{Be}_{22.5}$ (Vit1) and $\mathrm{Zr}_{58.5} \mathrm{Cu}_{15.6} \mathrm{Ni}_{12.8} \mathrm{Al}_{10.3} \mathrm{Nb}_{2.8}$ (Vit106a), which both exhibit a mismatch in the high- and low-temperature viscosities $[8,11]$. Moreover, they are excellent metallic glass formers, which, if processed containerlessly, allow access to the entire supercooled liquid region from the liquidus temperature $\left(T_{\text {liq }}\right)$ down to the glass transition temperature $\left(T_{g}\right)$. To access the transition kinetics, we take advantage of the time resolution available at the modern in situ synchrotron x-ray experiments. Combined with the state-of-art levitation technique of electrostatic levitation (ESL), we show that the transition mechanism here cannot be understood by a change in the local structure alone.

Master alloys of the investigated samples were prepared by mixing proper amounts of high-purity elements of each constituent alloyed in an arc-melting furnace under Ti-gettered pure (99.9999\%) Ar atmosphere. ESL samples $(\sim 100 \mathrm{mg})$ were prepared by remelting small portions of the master alloys under the same arc-melting condition. Structural studies of the alloys using in situ synchrotron $\mathrm{x}$-ray diffraction were performed at the PETRA III P07 beam line at DESY (Deutsches Elektronen-Synchrotron, Hamburg). The diffraction experiments were carried out in the transmission mode with an incoming photon energy of $100 \mathrm{keV}$ and a sample size of $2-3 \mathrm{~mm}$ in diameter. The diffracted intensity was recorded using a two-dimensional Perkin Elmer XRD 1621 detector. Owing to the high brilliance of the synchrotron, diffraction patterns could be taken continuously during heating or cooling of the levitated droplet where an exposure time of $0.5-1 \mathrm{~s}$ gave already sufficient statistics. The integrated intensity spectra over the entire Debye-Scherrer ring was obtained using the FIT2D software package [21], corrected by the dark current of the detector and the empty levitation chamber scattering.

Quasielastic neutron scattering (QNS) experiments were performed at the time-of-flight spectrometer TOFTOF at Heinz Maier-Leibnitz Zentrum in Munich. An incoming neutron wavelength of $4.4 \AA$ combined with a chopper speed of $18.000 \mathrm{rpm}$ gives an instrument energy resolution of about $100 \mu \mathrm{eV}$ and a momentum transfer $q$ range of $0.5-2.6 \AA^{-1}$ at zero energy transfer, i.e., up to the first structure factor maximum of the alloy melt $\left(\sim 2.6 \AA^{-1}\right)$. Under this configuration, both self-diffusion coefficients and structural relaxation times can be accurately determined on a microscopic time and length scale within a single measurement. Samples were also processed using ESL. The only difference compared to in situ synchrotron diffraction experiments was the larger sample size (up to
$6 \mathrm{~mm}$ in diameter) and the longer measurement time (1-2 $\mathrm{h}$ per temperature) in order to obtain sufficient data statistics.

Samples in the ESL were processed under high-vacuum conditions $\left(<1 \times 10^{-6}\right.$ mbar $)$. Levitated samples were heated and melted using two $75 \mathrm{~W}$ lasers operating at a wavelength of $808 \mathrm{~nm}$. The sample temperature was monitored with an integrated pyrometer in the laser unit, calibrated according to $T_{\text {liq }}$ of the alloy determined by differential scanning calorimetry (DSC) assuming a constant hemispherical emissivity $\epsilon$ in the liquid. The overall uncertainty in the calibrated temperature is about $\pm 10 \mathrm{~K}$ on the absolute scale. In order to investigate the kinetics of the possible structural changes in the undercooled melt, in situ diffraction was also performed during heating or cooling at defined rates. This was realized by a feedback-control loop that coupled the heating laser power with the pyrometer temperature. Figure 1(a) shows time-temperature profiles obtained when cooling Vit106a from equilibrium melt down to $T_{g}$ at cooling rates of $50 \mathrm{~K} / \mathrm{min}, 100 \mathrm{~K} / \mathrm{min}$, and at free radiative cooling. It can be seen that for experiments with defined cooling rates, the sample temperature was very well controlled.

Figure 1(b) shows the corresponding heat capacity-the emissivity ratio $c_{p} / \epsilon$ obtained from free radiative cooling as well as the normalized laser power input as a function of the temperature for the $100 \mathrm{~K} /$ min cooling rate. $c_{p} / \epsilon$ is derived by assuming pure radiative heat loss according to Stefan-Boltzmann law $[9,16]$. The heating laser power contains in principle the same information. To highlight this, the data were normalized by a function in the form of $P_{\text {laser }}^{\mathrm{fit}}=a T^{n}+b\left(T^{4}-T_{0}^{4}\right)$, with $a=19.51$, $b=1.8 \times 10^{-12}, n=-7,6 \times 10^{-3}, T_{0}=1107.8$, and $T$ being the temperature. Here, the first term represents the

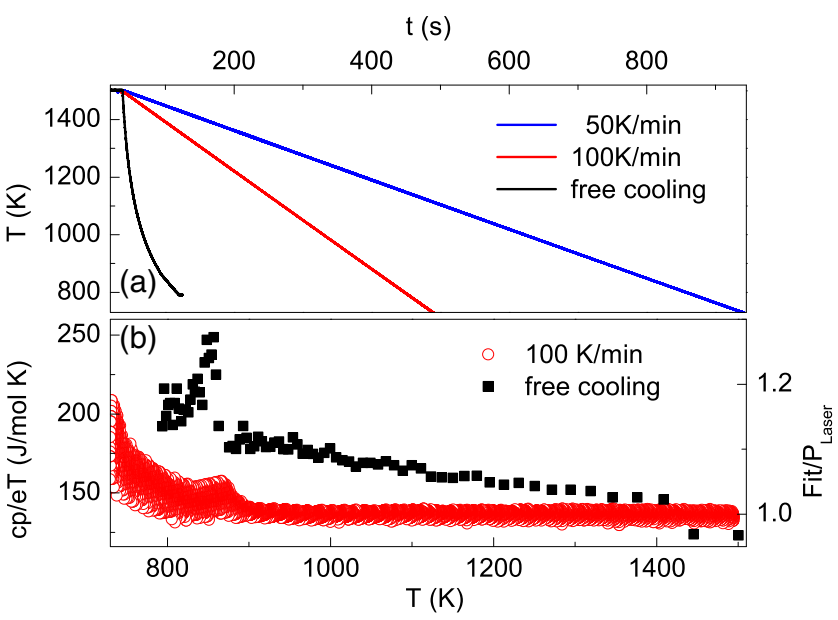

FIG. 1. ESL cooling experiments on Vit106a: (a) Time-temperature profiles for cooling rates of $50 \mathrm{~K} / \mathrm{min}, 100 \mathrm{~K} / \mathrm{min}$, and for free radiation cooling. (b) $c_{p} / \epsilon$ as derived from the free radiation cooling curve shown in (a). Normalized laser power of the $100 \mathrm{~K} / \mathrm{min}$ cooling rate as shown in (a). 


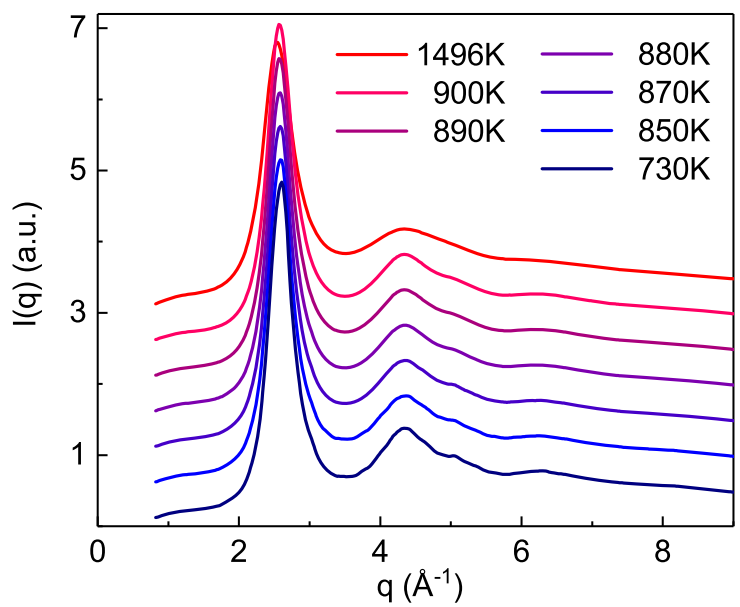

FIG. 2. Diffractograms of a Vit106a sample: Measured intensities as a function of the scattering vector $q$ when cooling at $100 \mathrm{~K} / \mathrm{min}$. Curves are shifted for clarity.

influence of the laser input while the second term represents the radiative losses.

The obtained heat capacity results here are in full agreement with the previous studies $[9,16]$. Assuming a constant emissivity $\epsilon$ of 0.22 allows us to derive $c_{p}$ on an absolute scale as measured by calorimetry [22]. In the undercooled melt which is not accessible by DSC, a sudden increase of the $c_{p} / \epsilon$ starting from $870 \pm 10 \mathrm{~K}$ can be observed, which is also visible at $888 \pm 7 \mathrm{~K}$ in the heating laser power for a cooling rate of $100 \mathrm{~K} / \mathrm{min}$. For a cooling rate of $50 \mathrm{~K} / \mathrm{min}$, this onset was found to be located at $890 \pm 5$ K. Similar features are observed on the Vit1 melt, consistent with the previous results [9].

Figure 2 shows the integrated intensity profiles as a function of momentum transfer $q$ from $\sim 1500 \mathrm{~K}$ down to $730 \mathrm{~K}$, measured at a cooling rate of $100 \mathrm{~K} / \mathrm{min}$. The diffractograms exhibit typical features of a disordered system, with broad minima and maxima. From the recorded diffraction patterns $I(q)$, the position of the first maximum $q_{\max }$ is obtained by interpolating the peak maximum in a small $q$ range using a polynomial of up to the third order. The position of the first structure factor maximum shifts to higher $q$ values with decreasing temperature, which is not surprising since the interatomic distance should decrease with decreasing temperature.

Sudden changes of the temperature-dependent first structure factor maximum in the undercooled melt associated with the maxima in $c_{p} / \epsilon$ have previously been observed for both alloy compositions $[9,16]$. As shown in Fig. 3, for both BMGs, $q_{\max }$ increases almost linearly when cooling before a discontinuity sets in. The discontinuity in $q_{\max }$ arises between 800 and $900 \mathrm{~K}$ for Vit106a and between 850 and $750 \mathrm{~K}$ for Vit1, which is consistent with the previous results observed during free radiative cooling $[9,16,23]$. For the obtained $q_{\max }$ with cooling rates of $50 \mathrm{~K} / \mathrm{min}, 100 \mathrm{~K} / \mathrm{min}$, and at free radiative cooling, the
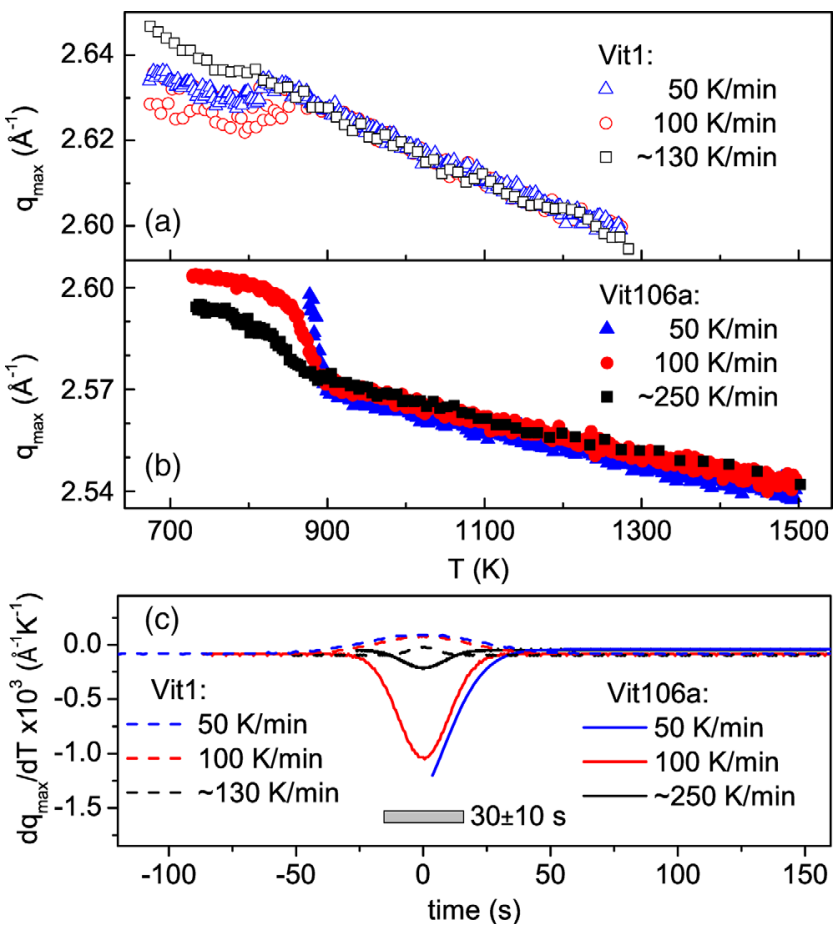

FIG. 3. Position of the first maximum of $S(q)\left(q_{\max }\right)$ as a function of the temperature. Cooling experiments on Vit1 (a) and Vit106a (b) when cooling rates of $50 \mathrm{~K} / \mathrm{min}, 100 \mathrm{~K} / \mathrm{min}$, and at free radiation cooling. (c) Timescale of the transition derived from the temperature course of $q_{\max }$ and cooling rate.

temperature dependence (slope) of $q_{\max }$ is almost independent of the cooling rate within the linear region. The onset temperatures obtained from thermodynamic and structural observations are summarized in Table I. They show consistently that they are associated with the same underlying LL-transition phenomena.

We also performed separately free radiative cooling experiments with much smaller samples to achieve larger cooling rates up to $400 \mathrm{~K} / \mathrm{min}$. There might be a weak cooling rate dependence of the onset temperature. Particularly, for Vit1 it seems that the onset temperature

TABLE I. Onset temperatures for structural changes $\left(T_{\text {onset }}^{q_{\max }}\right)$, thermal signals in the $c_{p} / \epsilon$ course $\left(T_{\text {onset }}^{c_{p} / \epsilon}\right)$, and laser power $\left(T_{\text {onset }}^{\text {laser }}\right)$ in Vit106a and Vit1 when cooling at $50 \mathrm{~K}, 100 \mathrm{~K}$, and free radiation cooling, which corresponds to cooling rates of approximately $250 \mathrm{~K} / \mathrm{min}$ at $876 \mathrm{~K}$ and $130 \mathrm{~K} / \mathrm{min}$ at $798 \mathrm{~K}$.

\begin{tabular}{lcccc}
\hline \hline Comp. & Cooling rate & $T_{\text {onset }}^{q_{\max }}(\mathrm{K})$ & $T_{\text {onset }}^{c_{p} / \epsilon}(\mathrm{K})$ & $T_{\text {onset }}^{\text {laser }}(\mathrm{K})$ \\
\hline Vit106a & Free cooling & $876( \pm 7.5)$ & $870( \pm 10.5)$ & $\ldots$ \\
& $100 \mathrm{~K} / \mathrm{min}$ & $892( \pm 10.5)$ & $\ldots$ & $888( \pm 7.5)$ \\
& $50 \mathrm{~K} / \mathrm{min}$ & $895( \pm 4.5)$ & $\ldots$ & $890( \pm 4.5)$ \\
Vit1 & Free cooling & $798( \pm 9.5)$ & $805( \pm 4)$ & $\ldots$ \\
& $100 \mathrm{~K} / \min$ & $841( \pm 10)$ & $\ldots$ & $815( \pm 7)$ \\
& $50 \mathrm{~K} / \min$ & $816( \pm 5)$ & $\ldots$ & $821( \pm 8)$ \\
\hline \hline
\end{tabular}


decreases with increasing cooling rate. However, this is still within the temperature uncertainty of the pyrometer. It should also be noted that the variation of the cooling or heating rate here is still less than an order of magnitude (from $\min 50 \mathrm{~K} / \min$ to $\max 400 \mathrm{~K} / \mathrm{min}$ ). Thus, this trend cannot be clearly identified.

In contrast, the temperature interval of the entire transition region-from the onset to the temperature where the original slope in $q_{\max }$ is recovered-increases with decreasing cooling rate. Transferring this interval across the LL transition to the transition time by taking the cooling rate into account, gives consistently $30 \pm 10 \mathrm{~s}$ for both the Vit1 and Vit106a melts. This is surprisingly slow considering that the melt dynamics are still not very sluggish at the transition temperature. It seems that long-range atomic transport is involved.

However, since both melts are multicomponent alloys, the change in $S(q)$ can neither be directly associated with the change of the interactomic distances nor with the macroscopic density which changes less than $0.1 \%$. Rather, it represents the sum of the contributions from the evolution of different partial structure factors across the LL transition, which cannot be disentangled here. Therefore, a positive or negative amplitude of $\Delta q_{\max }$ does not necessarily mean an increase or a decrease in the distance between a certain atomic pair. To be able to (semi-) quantitatively estimate transport distances, we investigated the self-diffusion coefficients of the alloys using QNS.

Figure 4 shows the measured self-diffusion coefficient and the structural relaxation time $\tau_{\max }$ at the structure factor maximum $q_{\max }$. The diffusion coefficient is derived from $D=1 /\left(\tau q^{2}\right)$ in the $q$ range between 0.5 and $1.2 \AA^{-1}$ where incoherent scattering is dominant [24]. In Vit1, this represents an average self-diffusion coefficient of $\mathrm{Ti}, \mathrm{Ni}$, and $\mathrm{Cu}$, and for Vit106a of $\mathrm{Cu}$ and $\mathrm{Ni}$, weighted with the

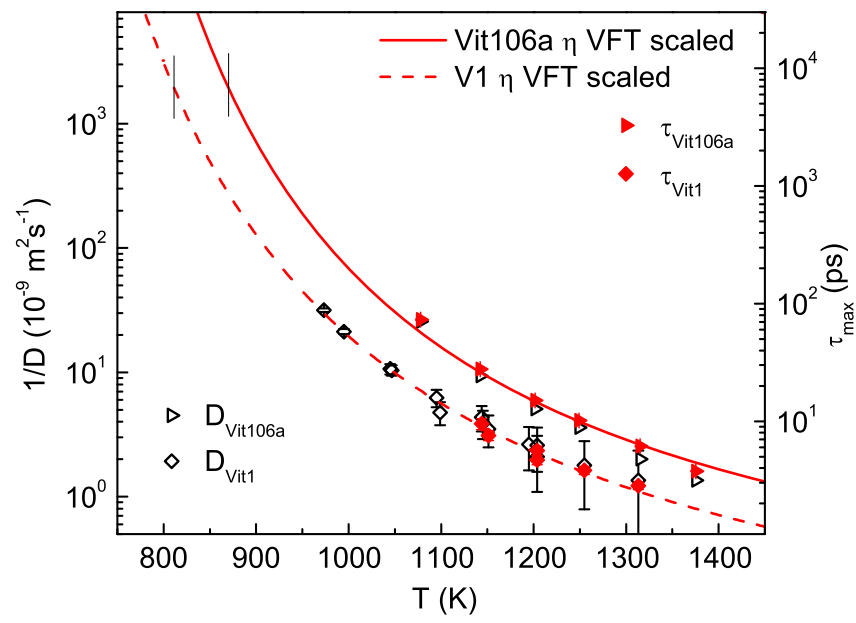

FIG. 4. The inverse average self-diffusion coefficient and the structural relaxation time of Vit1 and Vit106a in the same temperature range. Thin vertical lines show where the LL transition occurs. corresponding incoherent scattering cross sections. However, the value can be considered to be valid for all constituents within a factor of 2 [25]. It can be seen that $D$ follows the same temperature dependence of the structural relaxation time, which is almost identical to that of the melt viscosity [Vogel-Fulcher-Tammann- (VFT) like; see lines in Fig. 4] [25].

Considering only the high-temperature (fragile) liquid region, extrapolating the VFT temperature dependence of the melt viscosity yields the self-diffusion coefficient at the mean onset temperature of the LL transition. It is about $3 \times 10^{-13} \mathrm{~m}^{2} \mathrm{~s}^{-1}$ (at $885 \mathrm{~K}$ ) for Vit106a and $6 \times 10^{-13} \mathrm{~m}^{2} \mathrm{~s}^{-1}$ (at $810 \mathrm{~K}$ ) for Vit1. Assuming that the structural change is achieved by diffusion on a timescale of $30 \pm 10 \mathrm{~s}$, the diffusion length $l=\sqrt{6 D t}$ involved in the LL transition is estimated to be about 7-10 $\mu \mathrm{m}$. Taking into account the uncertainties originating from the temperature extrapolation and from the dynamic decoupling in the supercooled liquid region, we can assume that the melt dynamics change by roughly 1 to 2 orders of magnitude across the LL transition $[8,14]$. Still, the minimum $l$ would be on the order of $100 \mathrm{~nm}$. This confirms that during the LL transition, long-range atomic transport is obviously necessary.

In other glass formers like oxide or molecular melts, the microscopic mechanisms of LL transitions have been proposed based on the change of a local structure unit or environment: e.g., in the case of water, a two competing H-bonding environment [26], or in tetrahedral liquids, a different ordering [3-5,27], which is often accompanied by different densities of the involved phases. Here we found neither a significant density change across the LL transition $[9,16]$, nor were the slow transition kinetics compatible with a change of the local structural change alone. Nevertheless, similar structural manifestations have been observed in the LL transition of an $\mathrm{Al}_{2} \mathrm{O}_{3}-\mathrm{Y}_{2} \mathrm{O}_{3}$ melt [2], where domains of different liquids on the nanometer or even micrometer scale are involved.

As mentioned in the case of metallic melts, so far, only changes in the medium-range order are commonly considered to be relevant. However, taking the very large diffusion length into account, we cannot exclude here the possibility of involving different melt compositions, e.g., originated from chemical decomposition. This should be experimentally detectable via, e.g., small angle scattering techniques or resistivity measurements $[28,29]$. Although particularly in the case Vit1, spinodal decomposition in the glass has been reported, the results are still controversial and are observed at much lower temperatures [30-32].

Our observation of the rather slow transition kinetics also indicates that the intermediate states of the transition might be quenched into glass if the cooling rates are sufficiently high. This will allow the study of different stages of the transition in real space and element specifically, employing, e.g., advanced electron microscopies. Referring to the large number of alloy systems where LL transitions (strong to 
fragile) have been reported, and their significant impacts on the properties of the melt, our finding shows the need to consider different specific scenarios for the LL transition in BMGs, including its relation to chemical decomposition.

In conclusion, combining containerless processing techniques and in situ structural investigation, we show that the onset temperature of the LL transition in metallic glassforming liquids depends weakly on cooling rates on the order of $100 \mathrm{~K} / \mathrm{min}$. In contrast, the degree of the transition is sensitive to such temperature variations. Together with the melt dynamics, these features point to a transition mechanism which requires long-range transport of atoms in the melt, on the $100 \mathrm{~nm}$ lengthscale. Therefore, the LL transition in metallic glass-forming melts seems to be different compared to that in other liquids such as, e.g., water, where only the coordination or the bonding angle of the local structural units change. This might be due to the nondirectional bonding nature of the metallic melts, where not only the medium-range order but also different scenarios of the transition mechanism need to be considered.

We would like to thank the chair of metallic materials at the University Saarland for providing Vit106a and help during different experiments. We also thank Z. Evenson at TOFTOF, FRM II, U. Rütt and O. Gutowski at P07, PETRA III, DESY, as well as D. Holland-Moritz and C. C. Yuan from DLR-MP for their support during the synchrotron and neutron scattering experiments. We acknowledge DESY (Hamburg, Germany), a member of the Helmholtz Association HGF, for the provision of experimental facilities at PETRA III. We also acknowledge the access of the neutron scattering facilities on the TOFTOF instrument operated by TU München at the Heinz Maier-Leibnitz Zentrum, Garching, Germany. The financial support provided by the Deutsche Forschungsgemeinschaft through the Grant No. ME 1958/11-2 is gratefully acknowledged.

\section{*fan.yang@dlr.de}

[1] C. A. Angell, Science 319, 582 (2008).

[2] N. Greaves, M. Wilding, S. Fearn, D. Langstaff, F. Kargl, S. Cox, Q. V. Van, O. Majérus, C. Benmore, R. Weber et al., Science 322, 566 (2008).

[3] P. H. Poole, M. Hemmati, and C. A. Angell, Phys. Rev. Lett. 79, 2281 (1997).

[4] V. V. Vasisht, S. Saw, and S. Sastry, Nat. Phys. 7, 549 (2011).

[5] M. H. Bhat, V. Molinero, E. Soignard, V. C. Solomon, S. Sastry, J. L. Yarger, and C. A. Angell, Nature (London) 448, 787 (2007).

[6] C. Zhang, L. Hu, Y. Yue, and J. Mauro, J. Chem. Phys. 133, 014508 (2010).
[7] Q. S. Zeng, Y. Ding, W. L. Mao, W. Yang, S. V. Sinogeikin, J. Shu, H. K. Mao, and J.Z. Jiang, Phys. Rev. Lett. 104, 105702 (2010).

[8] Z. Evenson, T. Schmitt, M. Nicola, I. Gallino, and R. Busch, Acta Mater. 60, 4712 (2012).

[9] S. Wei, F. Yang, J. Bednarcik, I. Kaban, O. Shuleshova, A. Meyer, and R. Busch, Nat. Commun. 4, 2083 (2013).

[10] W. Xu, M. T. Sandor, Y. Yu, H.-B. Ke, H.-P. Zhang, M.-Z. Li, W.-H. Wang, L. Liu, and Y. Wu, Nat. Commun. 6, 7696 (2015).

[11] I. Jonas, W. Hembree, F. Yang, R. Busch, and A. Meyer (to be published).

[12] R. Kurita and H. Tanaka, Science 306, 845 (2004).

[13] F. Perakis, K. Amann-Winkel, F. Lehmkühler, M. Sprung, D. Mariedahl, J. A. Sellberg, H. Pathak, A. Späh, F. Cavalca, D. Schlesinger et al., Proc. Natl. Acad. Sci. U.S.A. 114, 8193 (2017).

[14] C. Way, P. Wadhwa, and R. Busch, Acta Mater. 55, 2977 (2007).

[15] S. Wei, M. Stolpe, O. Gross, Z. Evenson, I. Gallino, W. Hembree, J. Bednarcik, J. J. Kruzic, and R. Busch, Appl. Phys. Lett. 106, 181901 (2015).

[16] M. Stolpe, I. Jonas, S. Wei, Z. Evenson, W. Hembree, F. Yang, A. Meyer, and R. Busch, Phys. Rev. B 93, 014201 (2016).

[17] S. Küchemann and K. Samwer, Acta Mater. 104, 119 (2016).

[18] J. Li, W. K. Rhim, C. P. Kim, K. Samwer, and W. L. Johnson, Acta Mater. 59, 2166 (2011).

[19] J. C. Bendert, M. E. Blodgett, A. K. Gangopadhyay, and K. F. Kelton, Appl. Phys. Lett. 102, 211913 (2013).

[20] A. C. Barnes, L. B. Skinner, P. S. Salmon, A. Bytchkov, I. Pozdnyakova, T. O. Farmer, and H. E. Fischer, Phys. Rev. Lett. 103, 225702 (2009).

[21] A. Hammersley, FIT2D V9.129 reference manual V3.1, ESRF Inter Report No. ESRF98HA01, 1998.

[22] I. Gallino, M. Shah, and R. Busch, Acta Mater. 55, 1367 (2007).

[23] The cooling rates are slightly different as indicated here due to different sample sizes.

[24] A. Meyer, W. Petry, M. Koza, and M. P. Macht, Appl. Phys. Lett. 83, 3894 (2003).

[25] F. Yang, T. Unruh, and A. Meyer, Europhys. Lett. 107, 26001 (2014).

[26] E. A. Jagla, J. Phys. Condens. Matter 11, 10251 (1999).

[27] H. Tanaka, J. Phys. Condens. Matter 15, L703 (2003).

[28] D. P. B. Aji and G. P. Johari, J. Chem. Phys. 148, 144506 (2018).

[29] G. Lohöfer, Meas. Sci. Technol. 16, 417 (2005).

[30] S. Schneider, P. Thiyagarajah, U. Geyer, and W. L. Johnson, Physica (Amsterdam) (241-243)B, 918 (1997).

[31] J. F. Löffler and W. L. Johnson, Appl. Phys. Lett. 76, 3394 (2000).

[32] I. Martin, T. Ohkubo, M. Ohnuma, B. Deconihout, and K. Hono, Acta Mater. 52, 4427 (2004). 